DESY 93-139

SCIPP $93 / 35$

November 1993

\title{
Dijet Production at Large Rapidity Intervals
}

\author{
Vittorio Del Duca \\ Deutsches Elektronen-Synchrotron \\ DESY, D-22607 Hamburg , GERMANY \\ and \\ Carl R. Schmidt 円 \\ Santa Cruz Institute for Particle Physics \\ University of California, Santa Cruz, CA 95064, USA
}

\begin{abstract}
We examine dijet production at large rapidity intervals at Tevatron energies, by using the theory of Lipatov and collaborators which resums the leading powers of the rapidity interval. We analyze the growth of the Mueller-Navelet $K$-factor in this context and find it to be negligible. However, we do find a considerable enhancement of jet production at large transverse momenta. In addition, we show that the correlation in transverse momentum and azimuthal angle of the tagging jets fades away as the rapidity interval is increased.
\end{abstract}

\footnotetext{
${ }^{1}$ Supported in part by the U.S. Department of Energy.
} 


\section{Introduction}

As the search for the top quark continues, the Tevatron Collider continues to produce large amounts of data on hadronic jets [1]. This data provides a unique opportunity to test our understanding of jet production at high energies. The calculation of jet production rates at hadron colliders is a challenging problem of perturbative QCD, because it involves many different scales, including: $\Lambda_{Q C D}$, the hadron-hadron center-of-mass energy $\sqrt{s}$, the parton-parton center-of-mass energy $\sqrt{\hat{s}}$, and the momentum transfer $Q$, which is of the order of the transverse momentum of the jets produced in the hard scattering.

The conventional approach to these calculations is to work at fixed order in the coupling constant $\alpha_{s}$, assuming that $\sqrt{s}, \sqrt{\hat{s}}$, and $Q$ are comparable in size, so that there are no large logarithms involving them. The effects of $\Lambda_{Q C D}$ are factorized into the parton structure functions, which are then evaluated at a scale of order $Q$ using the usual DGLAP evolution. At present the first radiative corrections to the Born processes are available [2]. These yield a more detailed description of the jet structure, reduce the dependence on the factorization scale, and are in very good agreement with the data on the one jet inclusive distribution at large transverse momenta [3].

At the high energies of the Tevatron, however, there may be kinematic configurations where one cannot ignore the effects of the disparate energy scales. In the semihard region, defined as $s>>Q^{2}>>\Lambda_{Q C D}^{2}$, the calculation of jet cross sections is characterized by the appearance of coefficients containing logarithms of large ratios of the kinematical invariants. If no restrictions are made on $\hat{s}=x_{1} x_{2} s$, these logarithms will involve the small- $x$ behavior of the structure functions, requiring a more sophisticated analysis than the usual DGLAP evolution [4]. Combined with the experimental uncertainties in the structure functions at small- $x$, it appears very difficult to make precise predictions in this kinematic region.

One way to overcome this problem is to try to disentangle the different ratios of kinematic invariants in the process. This can be achieved, for example, by requiring that the parton momentum fractions, $x_{1}$ and $x_{2}$, are large enough that no large ratios, other than the usual $Q^{2} / \Lambda_{Q C D}^{2}$, appear in the evolution of the parton distribution functions [5]. The price to be paid is that the logarithms of the kind $\hat{s} / Q^{2}$ will now appear in the parton subprocess. These logarithms are of the size of the rapidity interval in the scattering process. To realize this configuration experimentally, Mueller and Navelet proposed to 
tag two jets at the extremes of the Lego plot in azimuthal angle and rapidity, at fixed $x_{1}$, $x_{2}$ and transverse momenta $p_{\perp}$, and to watch the growth of the dijet inclusive cross section as the rapidity interval between the tagging jets grew with the center-of-mass energy. To deal with the large logarithms they used the Balitsky-Fadin-Kuraev-Lipatov theory (BFKL) [6], which systematically resums the leading powers in the rapidity interval by using a multigluon amplitude, with the gluons uniformly filling the rapidity interval between the tagging jets. The Mueller-Navelet $K$-factor, defined as the ratio between the resummed and the Born dijet cross sections at large rapidity intervals and fixed $x$ 's, exhibits the power-like growth in the center-of-mass energy typical of the BFKL resummation.

In this paper we study the Mueller-Navelet dijet cross section at the Tevatron energy of $\sqrt{s}=1.8 \mathrm{TeV}$. Since $\sqrt{s}$ is fixed, we instead let $x_{1}$ and $x_{2}$ vary with the rapidity interval. At the same time we retain the Mueller-Navelet requirement that $x_{1}, x_{2}$ are large enough that the parton distribution functions can be described by the DGLAP evolution. This is done by tagging on the two jets at the extremes of the rapidity interval $y$ with transverse momenta larger than some cutoff $p_{\perp \text { min. }}$. For reasonable values of $y$ and $p_{\perp \text { min }}$ the momentum fractions $x_{1}, x_{2}$ will be sufficiently large. We can then study the effects of the minijets in the BFKL resummation as a function of the kinematic variables of the two tagging jets.

In the exposition of this paper we follow the outline of Ref. [7]. Namely, in sect. 2 we consider the inclusive dijet production $p p \rightarrow 2$ jets $+X$ at the Born-level, both exact [8] and in the large-rapidity limit. We then compute the leading logarithmic corrections at large rapidity as done in Ref. [5]. In section 3 we present numerical results for the inclusive dijet production, with and without integrating over the jet transverse momenta. We discuss the effects of the BFKL resummation on the growth of the Mueller-Navelet $\mathrm{K}$-factor, on the single jet $p_{\perp}$ distribution at large $y$, and on the correlations of the two jets in transverse momentum and azimuthal angle as the rapidity interval is increased. In section 4 we include some remarks on the range of validity and the limitations of this purely leading logarithmic calculation, and we present our conclusions.

\section{The Dijet Inclusive Cross section}

We are going to study the semi-inclusive process $p_{A} p_{B} \rightarrow 2$ jets $+X$ in the semihard regime defined by $\hat{s}>>Q^{2}$, with $Q^{2}$ being a typical momentum scale in the event, 
$Q^{2} \approx p_{1 \perp} p_{2 \perp}$. The two tagged jets are chosen with a large rapidity interval $y=y_{1}-y_{2} \approx$ $\ln \left(\hat{s} / p_{1 \perp} p_{2 \perp}\right)$. Other relevant parameters in the event are the relative azimuthal angle $\phi$ and the rapidity boost $\bar{y}=\left(y_{1}+y_{2}\right) / 2$ of the two jets.

In the semihard, large- $y$ regime we can write the cross section:

$$
\frac{d \sigma}{d p_{1 \perp}^{2} d p_{2 \perp}^{2} d \phi d y d \bar{y}}=\sum_{i j} x_{1} x_{2} f_{i / A}\left(x_{1}, \mu^{2}\right) f_{j / B}\left(x_{2}, \mu^{2}\right) \frac{d \hat{\sigma}_{i j}}{d p_{1 \perp}^{2} d p_{2 \perp}^{2} d \phi}
$$

with $f_{i(j)}=Q, \bar{Q}, G$ labeling the structure function of the parton species and flavor $i(j)=$ $q, \bar{q}, g$ inside hadron $A(B)$. The parton subprocess cross section $d \hat{\sigma}_{i j} / d p_{1 \perp}^{2} d p_{2 \perp}^{2} d \phi$ contains the sum over all additional particles (i.e. minijets) in the event. The factorization of the minijets into the subprocess cross section is possible, because at large $y$ the initial parton momentum fractions $x_{1}$ and $x_{2}$ are fixed in terms of the two tagged jet momenta, and are essentially independent of the particles filling the rapidity interval. We will arrive at this cross section in several steps, starting with the exact Born level cross section, taking it to the $y>>1$ limit, and finally filling in the rapidity interval with the minijets.

i) Born Level Cross section. At the Born level the two partonic jets are produced back-to-back. The exact lowest order cross section can be put in the form (1) with the replacement

$$
\frac{d \hat{\sigma}_{i j}}{d p_{1 \perp}^{2} d p_{2 \perp}^{2} d \phi} \Rightarrow \frac{d \hat{\sigma}_{i j}}{d \hat{t}} \delta\left(p_{1 \perp}^{2}-p_{2 \perp}^{2}\right) \delta(\phi-\pi)
$$

The parton momentum fractions and the subprocess invariants at this level are given by:

$$
\begin{aligned}
x_{1} & =\frac{2 p_{\perp} e^{\bar{y}}}{\sqrt{s}} \cosh (y / 2) \\
x_{2} & =\frac{2 p_{\perp} e^{-\bar{y}}}{\sqrt{s}} \cosh (y / 2) \\
\hat{s} & =x_{1} x_{2} s=2 p_{\perp}^{2}(1+\cosh (y)) \\
\hat{t} & =-p_{\perp}^{2}\left(1+e^{-y}\right) \\
\hat{u} & =-p_{\perp}^{2}\left(1+e^{y}\right),
\end{aligned}
$$

where $p_{\perp}=p_{1 \perp}=p_{2 \perp}$. The subprocess invariants do not depend on the rapidity boost $\bar{y}$. This is a general property, since $\bar{y}$ parametrizes the collective motion of the parton subprocess in the hadron reference frame. The lowest order parton cross sections are well known and can be found in, for instance, Ref. [8]. 
ii) Large- $y$ Born Cross section. We now investigate the lowest order cross section when the rapidity interval $y$ is large. For $y>>1$ the lowest order amplitude is dominated by diagrams with gluon-exchange in the $t$-channel as in Fig. 1(a). In this limit the only subprocesses that contribute are $g g \rightarrow g g$ and $q g \rightarrow q g$ and $q q \rightarrow q q$. We obtain

$$
\frac{d \hat{\sigma}_{g g}}{d \hat{t}}=\frac{\pi C_{A}^{2} \alpha_{s}^{2}}{2 p_{\perp}^{4}}
$$

with $C_{A}=N_{c}=3$ the Casimir operator of the adjoint representation. Similarly, we find

$$
\frac{d \hat{\sigma}_{q q}}{d \hat{t}}=\frac{C_{F}}{C_{A}} \frac{d \hat{\sigma}_{q g}}{d \hat{t}}=\frac{C_{F}^{2}}{C_{A}^{2}} \frac{d \hat{\sigma}_{g g}}{d \hat{t}},
$$

with $C_{F}=\left(N_{c}^{2}-1\right) / 2 N_{c}=4 / 3$ the Casimir operator of the fundamental representation. Thus, it suffices to consider the subprocess $g g \rightarrow g g$ and include the other subprocesses by means of the effective structure function [9]

$$
f_{e f f}\left(x, \mu^{2}\right)=G\left(x, \mu^{2}\right)+\frac{C_{F}}{C_{A}} \sum_{f}\left[Q_{f}\left(x, \mu^{2}\right)+\bar{Q}_{f}\left(x, \mu^{2}\right)\right],
$$

where the sum is over the quark flavors. The parton momentum fractions in the large- $y$ limit are

$$
\begin{aligned}
& x_{1}=\frac{p_{1 \perp}}{\sqrt{s}} e^{(\bar{y}+y / 2)}=\frac{p_{1 \perp}}{\sqrt{s}} e^{y_{1}} \\
& x_{2}=\frac{p_{2 \perp}}{\sqrt{s}} e^{(-\bar{y}+y / 2)}=\frac{p_{2 \perp}}{\sqrt{s}} e^{-y_{2}} .
\end{aligned}
$$

Equation (『) is also valid in the large-y limit when higher-order corrections are included, so that $p_{1 \perp} \neq p_{2 \perp}$.

iii) Minijet-corrected Cross section. As discussed in the introduction, going to higher orders in the coupling constant, i.e. to multiple parton emission, we encounter large logarithmic contributions. In the semihard regime, the BFKL theory [6] systematically resums the leading logarithmic terms $\ln \left(\hat{s} / Q^{2}\right)$ by using a multigluon amplitude where the rapidity interval between the tagging jets is filled with gluons, strongly ordered in rapidity. This amplitude is shown in Fig. 1(b), where the thick line represents the resummation of the virtual radiative corrections, whose effect is to reggeize the gluons exchanged in the $t$ channel. The real gluons are inserted on these using the Lipatov effective three-gluon vertex [6]. The BFKL multigluon amplitude is then put in a rapidity-ordered 
phase space, the rapidities of the gluons are integrated out, and the dependence of the cross section on the gluon transverse momenta is reduced to the resolution of an integral equation. Its solution is then convoluted with a jet emission vertex on each side of the rapidity interval to give the minijet-corrected parton cross section for two jets at large- $y$ :

$$
\frac{d \hat{\sigma}_{g g}}{d^{2} p_{1 \perp} d^{2} p_{2 \perp}}=\left[\frac{C_{A} \alpha_{s}}{p_{1 \perp}^{2}}\right] f\left(y, p_{1 \perp}, p_{2 \perp}\right)\left[\frac{C_{A} \alpha_{s}}{p_{2 \perp}^{2}}\right] .
$$

In this equation $f\left(y, p_{1 \perp}, p_{2 \perp}\right)$ is the Laplace transform in the rapidity interval $y$,

$$
f\left(y, p_{1 \perp}, p_{2 \perp}\right)=\int \frac{d \omega}{2 \pi i} e^{\omega y} f_{\omega}\left(p_{1 \perp}, p_{2 \perp}\right),
$$

of the solution of the BFKL integral equation

$$
f_{\omega}\left(p_{1 \perp}, p_{2 \perp}\right)=\frac{1}{(2 \pi)^{2}} \sum_{n=-\infty}^{\infty} e^{i n(\phi-\pi)} \int_{-\infty}^{\infty} d \nu \frac{\left(p_{1 \perp}^{2}\right)^{-1 / 2+i \nu}\left(p_{2 \perp}^{2}\right)^{-1 / 2-i \nu}}{\omega-\omega(n, \nu)} .
$$

The eigenvalue of the integral equation $\omega(n, \nu)$ is

$$
\omega(n, \nu)=\frac{2 C_{A} \alpha_{s}}{\pi}\left[\psi(1)-\operatorname{Re} \psi\left(\frac{|n|+1}{2}+i \nu\right)\right]
$$

with $\psi$ the logarithmic derivative of the Gamma function. Substituting (9) and (10) in (8), and doing the integral over $\omega$, the minijet-corrected parton cross section becomes

$$
\frac{d \hat{\sigma}_{g g}}{d p_{1 \perp}^{2} d p_{2 \perp}^{2} d \phi}=\frac{C_{A}^{2} \alpha_{s}^{2}}{4 \pi p_{1 \perp}^{3} p_{2 \perp}^{3}} \sum_{n} e^{i n(\phi-\pi)} \int_{0}^{\infty} d \nu e^{\omega(n, \nu) y} \cos \left(\nu \ln \frac{p_{1 \perp}^{2}}{p_{2 \perp}^{2}}\right) .
$$

If we integrate over the azimuthal angle $\phi$ in (12), only the $n=0$ term survives.

iv) Minijet-corrected Cross section in the Saddle Point Approximation. At very large values of the rapidity interval $y$, the correlations between the two jets are washed out by the random walk in transverse momentum space of the gluons exchanged in the $t$ channel. This can be seen most easily by evaluating (12) in the saddle-point approximation. The contribution of (11) to this equation is dominated by $n=0$ and is strongly peaked near $\nu=0$. Thus we keep only the first term in the Fourier expansion in $\phi$, and expand $\omega(\nu)=\omega(0, \nu)$ about $\nu=0$

$$
\omega(\nu)=A-B \nu^{2}+\cdots
$$


with

$$
A=\frac{4 C_{A} \alpha_{s}}{\pi} \ln 2, \quad B=\frac{14 C_{A} \alpha_{s}}{\pi} \zeta(3) .
$$

Then we can evaluate (12) using the saddle-point approximation for the integral over $\nu$, to obtain

$$
\frac{d \hat{\sigma}_{g g}}{d p_{1 \perp}^{2} d p_{2 \perp}^{2} d \phi}=\frac{C_{A}^{2} \alpha_{s}^{2}}{8 p_{1 \perp}^{3} p_{2 \perp}^{3}} \frac{e^{A y}}{\sqrt{B \pi y}} \exp \left(-\frac{\ln ^{2}\left(p_{1 \perp}^{2} / p_{2 \perp}^{2}\right)}{4 B y}\right) .
$$

The exponential growth of (15) with the rapidity interval $y$ is due to the production of the minijets.

\section{$3 \quad$ Numerical Results}

We now examine numerically the effects of the minijets at the Tevatron center-of-mass energy $\sqrt{s}=1.8 \mathrm{TeV}$. We are mainly interested in understanding the behavior of the parton subprocess, which does not depend on $\bar{y}$. Therefore, except where indicated, we work at fixed $\bar{y}$ and observe the cross sections as a function of the rapidity interval $y$. We chose $\bar{y}=0$ so that neither $x$ can become too small. For consistency of notation we will refer to the leading jet in rapidity as jet 1 and the trailing jet as jet 2 (i.e., $\left.y_{1}=+|y| / 2, y_{2}=-|y| / 2\right)$. Of course, everything is symmetric under the exchange of the two jets. We have used the leading order CTEQ structure functions [10] with the renormalization and factorization scale set to the geometric mean of the transverse momenta of the tagging jets, $\mu^{2}=p_{1 \perp} p_{2 \perp}$. We shall address some of the difficulties involved in the choice of scale in the next section. For $p_{\perp \text { min }}>10 \mathrm{GeV}$ and $\bar{y}=0$ the parton density functions are always evaluated at $x>10^{-2}$, so we are justified in using the DGLAP evolution in this region of phase space.

We begin by looking for the exponential growth of equation (15) in the cross section as originally suggested by Mueller and Navelet. To do this we integrate over the azimuthal angle $\phi$ and over both transverse momenta above a cutoff of $p_{\perp \min }=20 \mathrm{GeV}$. In Fig. 2 (a) we present this cross section in the first three approximations $(i$-iii) of section 2 . From the plot we see that the large- $y$ Born cross section is a good approximation to the exact Born level cross section for large $y \gtrsim 4$. However, the minijet-corrected cross section does not exhibit any great enhancement at large rapidity. This is more easily seen in a plot of the K-factor, defined here as the ratio of the minijet-corrected cross section to 
the large- $y$ Born cross section,

$$
K=\frac{d \sigma(\text { minijet })}{d y d \bar{y}} / \frac{d \sigma(\text { large }-y)}{d y d \bar{y}} .
$$

The $K$-factor is defined so that $K \rightarrow 1$ as $y \rightarrow 0$. In Fig. 2(b) we see that the $K$-factor increases until $y \approx 6$, but then quickly goes to zero 2 .

This effect can be understood if we remember that the rapidity dependence enters not only in the BFKL kernel $f\left(y, p_{1 \perp}, p_{2 \perp}\right)$, but also in the parton structure functions $f_{i}\left(x_{1}, \mu^{2}\right) f_{j}\left(x_{2}, \mu^{2}\right)$ where the momentum fractions are given by (7). The allowed phase space in $p_{1 \perp}, p_{2 \perp}$ is substantially decreased at large $y$ by the restriction that the momentum fractions must be less than 1 . The decrease in phase space has a greater effect on the minijet cross section, with the result that the $K$-factor is cut off at large $y$. It requires a much larger range in scales from $p_{\perp \text { min }}$ to $\sqrt{s}$ in order to approach the exponential growth of the Mueller-Navelet $K$-factor. This is exhibited in Fig. 3 where we show the $K$-factor at the Tevatron energy, at a Large Hadron Collider energy of $\sqrt{s}=15 \mathrm{TeV}$, and at $\sqrt{s}=10^{5} \mathrm{TeV}$. The full exponential growth is achieved in the limiting case of an infinitely large value of $\sqrt{s}$, where the cutoff in the phase space of the minijet cross section never occurs.

Thus, at the Tevatron energy we must look elsewhere for effects of the minijets. In Fig. 4(a) we show the $p_{\perp}$ distributions of jet 1 for a rapidity interval of $y=4$. We plot the minijet cross section with two different cutoffs for the second jet, $p_{2 \perp \text { min }}=10 \mathrm{GeV}$ and $20 \mathrm{GeV}$, while the Born level cross section always has $p_{2 \perp}=p_{1 \perp}$. These plots exhibit two effects of the minijets. We see that the overall scale of the $p_{\perp}$ distribution depends strongly on the minimum $p_{\perp}$ of the second jet, and that the slope of the distribution is flatter with a substantial increase at large $p_{\perp}$. In Fig. 4(b) at $y=6$ we see an even greater dependence on $p_{2 \perp \text { min }}$. These effects can be partially understood by the fact that the $p_{\perp}$ of jet 1 can be balanced by the smaller $p_{\perp}$ of jet 2 and the minijets produced in the rapidity interval. The lower $x$ values required for this type of event increase its likelihood relative to the back-to-back dijet event, which is all that can occur at the Born level. It is even possible for jet 1 to attain transverse momenta that are kinematically impossible at the Born level.

These arguments suggest that the minijets occuring in the rapidity interval between the tagged jets will cause the tagged jets to become uncorrelated. This can be seen easily

\footnotetext{
${ }^{2}$ The fact that $K$ goes below 1 for small $y$ is presumably a computational artifact, arising from the difficulty in doing the numerical integration over a very sharply peaked function at small $y$.
} 
in the minijet formulae by looking at the BFKL kernel as it is varied from $y=0$ to very large $y$. For very small rapidities we approach the Born cross section (田) with

$$
f\left(y, p_{1 \perp}, p_{2 \perp}\right) \rightarrow \delta\left(p_{1 \perp}^{2}-p_{2 \perp}^{2}\right) \delta(\phi-\pi) .
$$

The two jets are produced back-to-back in $p_{\perp}$ and $\phi$. However, as the rapidity interval becomes large, we have

$$
f\left(y, p_{1 \perp}, p_{2 \perp}\right) \rightarrow \sim\left(p_{1 \perp} p_{2 \perp}\right)^{-1}
$$

and the tagging jets become completely uncorrelated.

The disappearence of correlations as $y$ increases can be seen dramatically in Fig. 5(a) where we plot the transverse momentum distribution of jet 1 at a fixed value of $p_{2 \perp}=50$ $\mathrm{GeV}$. For a rapidity interval of $y=2$ the cross section is strongly peaked near $p_{1 \perp}=p_{2 \perp}$. As the rapidity is increased there is a diffusion of the jet 1 momentum away from the jet 2 momentum until the peak is practically gone for $y=5$. In practice jet 2 will be integrated over some range of transverse momenta, so in Fig. 5(b) we show the same plot with $p_{2 \perp}$ integrated from $50 \mathrm{GeV}$ to $55 \mathrm{GeV}$ and $|\bar{y}| \leq 0.5$. To retain the normalization we have divided this cross section by $5 \mathrm{GeV}$.

Similarly, there is also a reduction of the correlation in the azimuthal angle $\phi$ as the rapidity interval increases. This can be seen in Fig. 6 , where we show the $\phi$ distribution with both jets integrated from $p_{\perp \text { min }}=20 \mathrm{GeV}$. The $\phi$ distribution is normalized to the uncorrelated cross section $d \sigma / d y d \bar{y}$, so that the area under each curve is equal to 1 . As expected the correlation in $\phi$ decreases as we vary from $y=5$ to $y=7$. The decorrelation in $\phi$, however, is slower than the decorrelation in $p_{\perp}$, because the eigenvalue (11) of the BFKL integral equation is more strongly peaked in $\nu$ than in $n$. For example, at $y=5$ the tagging jets are not correlated any more in $p_{\perp}$, while they still show a considerable correlation in $\phi$.

\section{Discussion and Conclusions}

The BFKL analysis that we have been using is a leading logarithmic approximation. With this in mind we offer some caveats to our results and discuss which effects should survive in an exact calculation. First, we must state that any of our plots at very small $y$ are not expected to be very accurate. For $y \lesssim 2$ there is even a reasonable 
discrepancy between the large- $y$ Born Cross section $(i i)$ and the exact Born level cross section $(i)$. However, we expect that the trends as $y$ is increased should be apparent even at reasonably small values of the rapidity interval. In particular the decorrelations in $p_{\perp}$ and $\phi$ should definitely increase with $y$.

There are also several ambiguities in our calculation, arising from the fact that the BFKL analysis assumes little variation in the $p_{\perp}$ of the minijets. For instance, the rapidity variable used in the standard BFKL analysis is $Y=\ln \left(\hat{s} / Q^{2}\right)$ where $Q$ is some typical scale of the minijets. In our calculation we have chosen $Q^{2}=p_{1 \perp} p_{2 \perp}$ so that $Y=y$, the experimental rapidity. This should not make a significant difference at large $y$, but it does emphasize the fact that the approximation becomes less reliable when the transverse momenta of the tagging jets are not of similar size, and there arise large logarithms of order $\ln \left(p_{1 \perp} / p_{2 \perp}\right)$. In addition, the dependence on jet definition, cone size, and other variables are subleading in this analysis at large $y$.

For related reasons the proper renormalization/factorization scale $\mu$ at which the coupling constant is evaluated is not well-determined in our leading logarithmic analysis. As required for the BFKL solution, we have evaluated the coupling for all of the minijets at a single scale of order $Q$. Possible choices for $\mu^{2}$ are $p_{1 \perp} p_{2 \perp}$ (as we have used), $p_{1 \perp}^{2}, p_{2 \perp}^{2}$, or $\max \left(p_{1 \perp}^{2}, p_{2 \perp}^{2}\right)$. At the level of our approximation, all of these scales are equivalent, but in practice the choice of scale can make a reasonable difference in the slope of the $p_{\perp}$ spectrum [5. However, our main conclusions about the decorrelation in transverse momentum and azimuthal angle at large $y$, as well as the increase in the cross section at large $p_{\perp}$ and $y$ will not change.

Finally, it is interesting to imagine a comparison of our results with a fixed $\mathcal{O}\left(\alpha_{s}^{3}\right)$ calculation. The fixed order calculation includes only the effects of up to three parton jets, while the BFKL resummation includes the leading contributions of amplitudes containing an arbitrary number of parton jets. From naive estimates one might expect that $y \lesssim 6$ is not large enough to warrant the use of the full BFKL analysis and that a next-to-leading order calculation is quite sufficient. However, as we have seen, the kinematic phase space is greatly enhanced by the sharing of transverse momentum among the additional minijets. This suggests that it may be necessary to go beyond $\mathcal{O}\left(\alpha_{s}^{3}\right)$ at large rapidity intervals. Moreover, the BFKL approximation clearly predicts the main features of the multiple jet emission at large $y$ and readily suggests the experiments to look for them. It would be exciting to compare the predictions of the BFKL resummation

\footnotetext{
${ }^{3}$ This ambiguity will arise in any non-exact calculation involving multiple jets with disparate energies.
} 
given here, as well as the next-to-leading order calculations, against experiment.

\section{Acknowledgements}

We wish to thank Bj Bjorken, Jerry Blazey, Terry Heuring, Michael Peskin and Harry Weerts for useful discussions.

\section{References}

[1] CDF Collaboration, preprint Fermilab-Conf-93-204-E, Fermilab-Conf-93-201-E; D0 Collaboration, preprint Fermilab-Conf-93-050-E, Fermilab-Conf-93-047-E.

[2] R.K. Ellis and J.C. Sexton, Nucl. Phys. B269 (1986) 445.

[3] S.D. Ellis, Z. Kunszt and D.E. Soper, Phys. Rev. D40 (1989) 2188; Phys. Rev. Lett. 62 (1989) 726; Phys. Rev. Lett. 64 (1990) 2121; Phys. Rev. Lett. 69 (1992) 1496; Phys. Rev. Lett. 69 (1992) 3615.

S.D. Ellis preprint CERN-TH-6861/93.

F. Aversa, M. Greco, P. Chiappetta and J.Ph. Guillet, Phys. Lett. 210B (1988) 225; Phys. Lett. 211B (1988) 465; Nucl. Phys. B327 (1989) 105; Z. Phys. C46 (1990) 253; Phys. Rev. Lett. 65 (1990) 401; Z. Phys. C49 (1991) 459.

[4] J.C. Collins and R.K. Ellis, Nucl. Phys. B360 (1991) 3;

S. Catani, M. Ciafaloni and F. Hautmann, Nucl. Phys. B366 (1991) 135.

[5] A.H. Mueller and H. Navelet, Nucl. Phys. B282 (1987) 727.

[6] L.N. Lipatov, Sov. J. Nucl. Phys. 23 (1976) 338;

E.A. Kuraev, L.N. Lipatov and V.S. Fadin, Sov. Phys. JETP 44 (1976) 443; Sov. Phys. JETP 45 (1977) 199;

Ya.Ya. Balitsky and L.N. Lipatov, Sov. J. Nucl. Phys. 28 (1978) 822.

[7] V. Del Duca and C.R. Schmidt, preprint SLAC-PUB-6228, to appear in Phys. Rev. D.

[8] B.L. Combridge, J. Kripfganz and J. Ranft, Phys. Lett. 70B (1977) 234. 
[9] B.L. Combridge and C.J. Maxwell, Nucl. Phys. B239 (1984) 429.

[10] J. Botts et al., Phys. Lett. 304B (1993) 159.

\section{Figure captions}

Fig.1: Two jet production amplitude in the large- $y$ limit at (a) the Born level and (b) with minijet corrections.

Fig.2: (a) Inclusive dijet production at the Tevatron, as a function of the rapidity interval $y$. The dashed and dot-dashed lines are respectively the exact and large- $y$ Born cross sections, and the solid line is the minijet-corrected cross section. (b) The $K$-factor, i.e. the ratio of the minijet-corrected cross section to the large- $y$ Born cross section, as a function of the rapidity interval $y$. The kinematic parameters for both figures are described in the text.

Fig.3: $K$-factor as a function of the rapidity interval $y$ at different center-of-mass energies. The cutoff on integration over both transverse momenta is at $p_{\perp \min }=20 \mathrm{GeV}$. From bottom to top, the solid lines represent the $K$-factors at Tevatron energies, at LHC energies $(\sqrt{s}=15 \mathrm{TeV})$, and at $\sqrt{s}=10^{5} \mathrm{TeV}$.

Fig.4: $p_{\perp}$ distribution of jet 1 at (a) $|y|=4$ and (b) $|y|=6$. The dashed and dotdashed lines are respectively the $p_{\perp}$ distributions for the exact and the large- $y$ Born cross section, for which $p_{1 \perp}=p_{2 \perp}$. The solid lines are the $p_{\perp}$ distributions of jet 1 for the minijet-corrected cross section, with two different cutoffs for jet $2, p_{2 \perp \text { min }}=10 \mathrm{GeV}$ and $20 \mathrm{GeV}$. Notice that in (b) the dashed and dotdashed lines completely overlap.

Fig.5: $p_{\perp}$ distribution of jet 1 with the jet 2 transverse momentum (a) fixed at $50 \mathrm{GeV}$, and (b) integrated from $50 \mathrm{GeV}$ to $55 \mathrm{GeV}$. From top to bottom, the solid lines are the $p_{\perp}$ distributions for the minijet-corrected cross section at $|y|=2,3,4,5$ and 6 . In (b) the rapidity boost $\bar{y}$ is integrated over $|\bar{y}| \leq 0.5$.

Fig.6: $\phi$ distribution normalized to the uncorrelated cross section $d \sigma / d y d \bar{y}$. From top to bottom, relative to the peak, the solid lines are the $\phi$ distributions for the minijet-corrected cross section at $|y|=5,6$ and 7 . 
This figure "fig1-1.png" is available in "png" format from: http://arxiv.org/ps/hep-ph/9311290v1 
This figure "fig1-2.png" is available in "png" format from: http://arxiv.org/ps/hep-ph/9311290v1 
This figure "fig1-3.png" is available in "png" format from: http://arxiv.org/ps/hep-ph/9311290v1 
This figure "fig1-4.png" is available in "png" format from: http://arxiv.org/ps/hep-ph/9311290v1 
This figure "fig1-5.png" is available in "png" format from: http://arxiv.org/ps/hep-ph/9311290v1 
This figure "fig1-6.png" is available in "png" format from: http://arxiv.org/ps/hep-ph/9311290v1 